\title{
Design and Analysis of Distributed Embedded Systems using AADL - Application to the Precision Time Protocol
}

\author{
Mohamed Yassin Chkouri \\ National School of Applied Sciences \\ $2222 \mathrm{M}$ hannech II \\ 93000 Tetouan - Morocco
}

\author{
Marius Bozga \\ Verimag, Centre Equation \\ 2, avenue de Vignate \\ 38610 GIERES - France
}

\begin{abstract}
Prototyping distributed embedded system can be seen as a collection of many requirements covering many domains. System designers and developers need to describe both functional and nonfunctional requirements. Building distributed systems is a very tedious task since the application has to be verifiable and analyzable. Architecture Analysis and Design Language (AADL) provides adequate syntax and semantics to express and support distributed embedded systems.

This paper studies a general methodology for translating AADL thread component depending on the thread implementation into the BIP (Behavior Interaction Priority) language and for prototyping distributed applications using the Precision Time Protocol (PTP) for building and translating AADL systems into a distributed application using network communication protocol. This allows simulation of systems specified in AADL to fully assess system viability, to refine and to correct the behavior of the system using the BIP (Behavior Interaction Priority) toolset.
\end{abstract}

\section{Keywords:}

Architecture Analysis and Design Language, Modeling, Distributed Embedded System, Model Transformation, Simulation, Validation

\section{INTRODUCTION}

Building distributed embedded systems requires a stringent methodology and involves many tightly coupled steps, from early requirements capture (number of tasks, their interactions, nonfunctional attributes) to validation (feasibility of scheduling) down to implementation and testing. Prototyping distributed applications can be extremely useful in evaluating a design, and also in understanding the effect of different parameters on the performance of an application.

Designing distributed systems is an extremely complex task and demands more attention and rigorous methodology. The produced distributed systems have to conform to many stringent functional and non-functional requirements from multiple contexts such as space, avionics, etc. Ensuring all requirements and features becomes very hard if the whole system is hand-coded. Thus, the application code should preferably be generated automatically from a verifiable and analyzable model. This facilitates the work of developers, helps during the stage of code verification and speed up the development cycle. Besides, constructing a verifiable model from the application model using model transformation is simpler and safer than constructing this model from source code.

Architecture Description Languages (ADLs) have been proposed to support the development process of embedded real-time and distributed applications. Among the ADLs, AADL [2] is the Architecture Analysis and Design Language that allows the modeling of distributed, real-time and embedded systems. AADL was first introduced to model the software and hardware architectures in the avionics domain. AADL can be seen as a collection of many requirements covering many domains. System designers and developers need to describe both functional and non-functional requirements. These requirements must then be sorted and enforced at the deployment level. We will present the set of requirements that must be respected to build distributed systems.

We have shown in previous work [13, 12], how AADL systems can be automatically translated into BIP [6] (Behavior Interaction Priority), and analyzed using the BIP toolset. BIP is a language for the description and composition of components as well as associated tools for analyzing models and generating code on a dedicated middleware. The language provides a powerful mechanism for structuring interactions involving rendezvous and broadcast. The model construction methodology applied to AADL models opens the way for enhanced analysis and early error detection by using BIP verification techniques. Once the model has been generated, three model checking techniques for verification can be applied: Model checking by Aldebaran [9], model checking with observer and D-Finder tool [8].

In this paper, we present a general methodology for translating AADL thread component that represents the most important part in the AADL architecture depending on the thread implementation behavior. The result of this study must take into account four cases: thread without implementation, thread containing a sequence of subprograms calls, behavior of thread described in the external file and thread behavior using the annex behavior [1]. We present also the translation to prototype distributed applications using BIP 
and network communication protocol. We begin with a model built by the application designer, who maps its application entities onto a hardware architecture. Then, we use AADL into BIP tool to generate BIP model conforming to AADL semantics. Finally, we use a code generator to generate an executable model for each system with communication protocol.

To illustrate the translation from distributed AADL systems into BIP, we use Precision Time Protocol (PTP) as a case study. Based on our experience, we use the AADL to model PTP and its translation into BIP. This translation allows the simulation of distributed systems and the application of formal verification techniques, e.g., verification of properties, deadlock detection, etc. Using our tool, we were able to run, to debug and evaluate the case study in a native platform (PC) before deploying it on a distributed embedded platform.

Code generation of distributed embedded applications from models is not limited to AADL. In fact, distributed and high-integrity systems are probably the domain which has the most maturity. OCARINA [15] allows model manipulation, generation of formal models to perform scheduling analysis and generate distributed applications. OCARINA allows code generation from AADL descriptions to Ada. PolyORB [18] is a middleware toolset that provides distribution services through standard programming interfaces and communication protocols. However, the generated code from AADL does not take into account the annex behavior specifications [1].

This paper is organized as follows. Section 2 gives an overview of AADL. In Section 3, we explain the translation of the thread component depending on four cases and how to translate AADL systems into a distributed application using network communication protocol. In Section 4, we present a PTP case study and its deployment into a distributed application. Conclusions close the article in Section 5.

\section{OVERVIEW OF AADL}

\subsection{Generalities}

The SAE Architecture Analysis \& Design Language (AADL) [2] is a textual and graphical language used to design and analyze the software and hardware architecture of performance-critical realtime systems. It plays a central role in several projects such as Topcased [4], OSATE [3], etc.

A system modeled in AADL consists of application software mapped to an execution platform. Data, subprograms, threads, and processes collectively represent application software. They are called software components. Processor, memory, bus, and device collectively represent the execution platform. They are called execution platform components. Execution platform components support the execution of threads, the storage of data and code, and the communication between threads. Systems are called compositional components. They permit software and execution platform components to be organized into hierarchical structures with welldefined interfaces. Operating systems may be represented either as properties of the execution platform or can be modeled as software components.

\subsection{AADL Components}

2.2.1 Software Components. AADL has the following categories of software components: subprogram, data, thread and process.

A subprogram component represents an execution entry-point in the source text. Subprograms can be called from threads and from other subprograms. These calls are handled sequentially by the threads. The data component type represents a data type in the source text that defines a representation and interpretation for instances of data. A thread represents a sequential flow of control that executes instructions within a binary image produced from source text. A thread always executes within a process. A scheduler manages the execution of a thread. A process represents a virtual address space. Process components are an abstraction of software responsible for executing threads.

2.2.2 Hardware Components. Execution platform components represent hardware and software that is capable of scheduling threads, interfacing with an external environment, and performing communication for application system connections.

AADL processor components are an abstraction of hardware and software that is responsible for scheduling and executing threads. In other words, a processor may include functionality provided by operating systems. A device component represents an execution platform component that interfaces with the external environment. A device can interact with application software components through their ports. A bus components are used to describe all kinds of networks, buses, etc. A Memory components are used to represent any storage device: RAM, hard disk, etc.

2.2.3 Systems Component. A system is the toplevel component of the AADL hierarchy of components. A system component represents a composite component as an assembly of software and execution platform components. All subcomponents of a system are considered to be contained in that system.

\subsection{Connections}

A connection is a linkage that represents communication of data and control between components. This can be the transmission of control and data between ports of different threads or between threads and processor or device components. We gave more details about the connection types and how to translated into BIP in previous work [11].

\subsection{Annex Behavior Specification}

Behavior specifications [1] can be attached to AADL model elements using an annex. The behavioral annex describes a transition system attached to subprograms and threads. Behavioral specifications are defined by the following elements:

- State variables section declares typed identifiers. They must be initialized in the initialization section.

-States section declares automaton states.

- Transitions section defines transitions from a source state to a destination state. The transition can be guarded with events or boolean conditions. An action part can be attached to a transition.

\section{FROM AADL TO DISTRIBUTED IMPLEMENTATION USING BIP}

\subsection{The BIP Component Framework}

BIP (Behavior Interaction Priority) is a framework for modeling heterogeneous real-time components [6]. The BIP framework consists of a language and a toolset including a frontend for editing and parsing BIP programs and a dedicated platform for model validation. The platform consists of an Engine and software infrastructure for executing models. It allows state space exploration and provides access to model-checking tools of the IF toolset [10] such as Aldebaran [9], as well as the D-Finder tool [8]. This permits to validate 


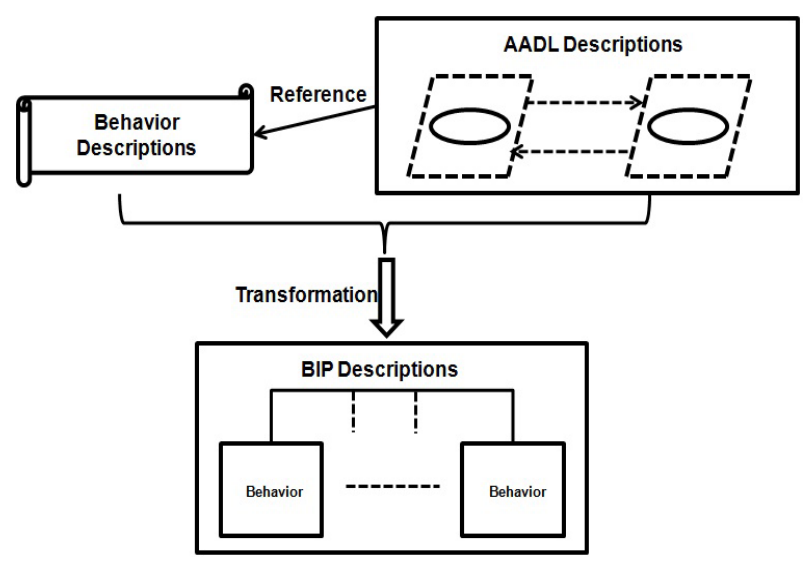

Fig. 1. Generation of the BIP application

BIP models and ensure that they meet properties such as deadlockfreedom, state invariants and schedulability. The BIP language allows hierarchical construction [14] of composite components from atomic ones by using connectors and priorities. Several case studies were carried out such as an MPEG4 encoder [17], TinyOS [7], and DALA [5].

\subsection{Transformation from AADL to BIP}

The AADL models are transformed into BIP automatically by using our AADL to BIP translation tool described in [12, 13]. Figure 1 shows the generation of an application from an AADL description that consists of translating AADL constructions by adding the behavior descriptions to BIP.

The model construction methodology applied to AADL models opens the way for enhanced analysis and early error detection by using BIP verification techniques. Once the model has been generated, three model checking techniques for verification can be applied:

3.2.1 D-Finder. is an interactive tool for checking deadlockfreedom for component-based systems by using a static analysis method. It takes as input BIP programs and applies proof strategies to eliminate potential deadlocks by computing increasingly stronger deadlocks.

3.2.2 Model checking by Aldebaran. The second technique of verification is model-checking by using the tool Aldebaran [9]. Exhaustive exploration by the BIP exploration engine generates a Labeled Transition System (LTS) which can be analyzed by model checking. For example, Aldebaran takes as input the LTS generated from BIP and checks for deadlock-freedom and other temporal properties.

3.2.3 Model checking with observers. The third technique of verification is by using BIP observers to express and check requirements. Observers allow us to express in a much simple manner most safety requirements. We apply this technique to verify some properties as verification of communication, and verification of thread deadline.

3.2.4 Simulation \& Debugging. In addition to the verification, we can simulate or test prototype implementations by creating an executable system. We can use an interactive simulation and debugger to verify each interaction step by step and to know which

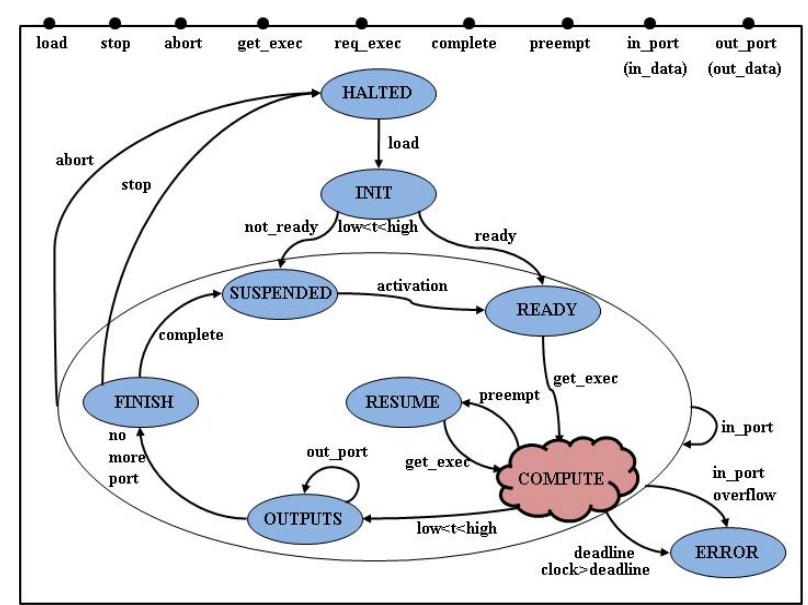

Fig. 2. BIP model for thread behavior

state or port is activated. These analysis allow to fully assess system viability, to refine and to correct the behavior of the system.

3.2.5 Code generator. The code generator takes as input a model, generated by the parser, and transforms it to a C++ application code. The application is an executable model of the original BIP program. Code is generated for each atomic component, connectors and priorities, i.e., the code is modular and preserves the structure of the initial model.

\subsection{Translation of Thread Component into BIP}

In this paper we present the translation of thread component that represents the principal component in the AADL architecture into BIP. This translation is the result of our study about this component. To have a complete translation of the thread component and to be able to use threads in larger domains, we must take into account four main cases of the threads implementation :

(1) without the implementation ;

(2) contains a sequence of subprograms calls ;

(3) contains annex behavior specification ;

(4) reference to a $\mathrm{C} / \mathrm{C}++$ file containing the behavior description.

The aim of this study about the thread behavior is to define general rules concerning the BIP code generation from AADL descriptions and the implementation of descriptions provided by the user.

An AADL thread is modelled in BIP by an atomic component as shown in Figure 2. The initial state of the thread is HALTED. On an interaction through port load the thread is initialized. Once initialization is completed the thread enters the READY state, if the thread is ready for an interaction through the port req_exec. Otherwise, it enters the SUSPENDED state. When the thread is in the SUSPENDED state it cannot be dispatched for execution.

When in the SUSPENDED state, the thread is waiting for an event and/or period to be activated depending on the thread dispatch protocol (periodic, aperiodic, sporadic). In the READY state, a thread is waiting to be dispatched through an interaction in the port get_exec. When dispatched, it enters the state COMPUTE to make a computation. Upon successful completion of the computation, the thread goes to the OUTPUTS state. If there are some outports to dispatch the thread returns to the OUTPUTS state. otherwise, it enters the FINISH state. 


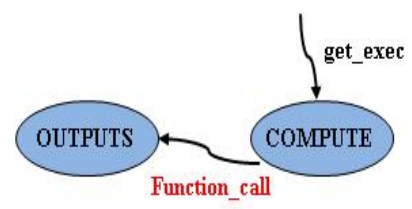

Fig. 3. Function call for the external file

The thread may be requested to enter its HALTED state through a port stop after completing the execution of a dispatch. A thread may also enter the thread HALTED state immediately through an abort port.

A thread without implementation provides no information about its internal structure. It is a type that has no implementation, or with empty behavior. So, they are non computation in the state COMPUTE. The translation of the AADL thread into BIP is shown in Figure 2.

3.3.1 Reference to an external file. In this case, the thread implementation is described in the external file using $\mathrm{C} / \mathrm{C}++$ programming language. The implementation is associated with the thread using AADL standard properties: Source_Name, Source_Text and Source Language as defined in the AADL standard.

The translation into BIP must link with the source code that the user must provide by matching the parameters. The user must write the source code implementation as a procedure or a function whose signature matches as described in AADL. The types of used data correspond to the types generated from the AADL data component. Figure 3 shows the translation of this case by replacing the state Compute in the Figure 2 by this one.

Figure 4 gives an example of AADL thread, called Sunseekerplant, it takes as input the float type Controllerinput and produce as output the float type Controllerinput. The implementation of the thread Sunseekerplant is described in an external $C$ file. It uses a function user_sunseekerplant in the file sunseekerplant.c.

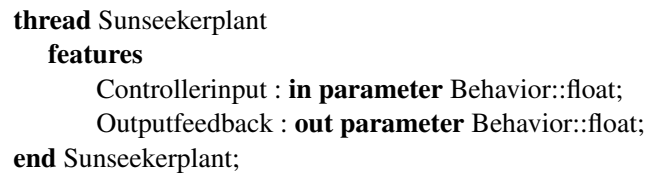

thread implementation Sunseekerplant.Beacon properties

Source_Language $\Rightarrow C$;

Source_Name $\Rightarrow$ " user_sunseekerplant ";

Source_Texe $\Rightarrow$ " sunseekerplant.c";

end Sunseekerplant.Beacon;

Fig. 4. Example of thread using external file as behavior

Figure 5 corresponds to the translation of the AADL thread to BIP. The link to the external file is described by a function call by matching the parameters of the thread during a transition from state COMPUTE to the state OUTPUTS through an interaction.

3.3.2 Using annex behavior specification. The implementation of thread behavior is described in this case by using the annex behavior specification [1]. The translation of AADL thread to BIP using behavior annex is described in Figure 6. As the figure shows, the behavior is always connected to the state COMPUTE to allow it to interact on the port preempt to access on the state RESUME.

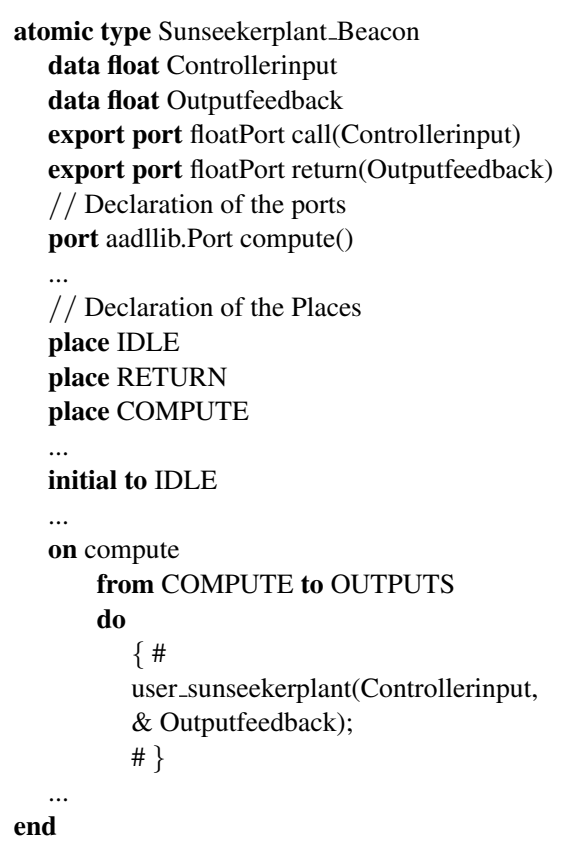

Fig. 5. Translation of the AADL thread to BIP

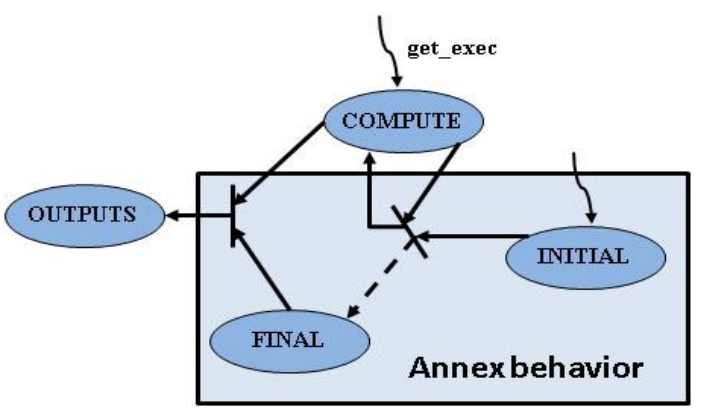

Fig. 6. BIP model for annex behavior

3.3.3 Using sequence of subprograms calls. In this case, we consider that the behavior of the thread consists of executing the call sequence of subprograms. A call to the subprograms in the AADL thread implementation is modeled in BIP as shown in Figure 7. This figure shows that the thread calls a subprogram through the port call, which expresses the call and send of the parameters to the subprogram and the return port expresses the end of the execution and the return of the parameters of the subprogram.

\subsection{Prototyping Distributed Implementation}

Building distributed systems is a very tedious task since the application has to be verifiable and statically analyzable. The AADL fits these two requirements and allows the designer to describe different aspects of his distributed application (number of processors, number of threads in each processors, connection between threads...). We begin with a model built by the application designer, who maps his application entities onto a hardware architecture. Then, we use AADL into BIP tool to generate BIP model conforming to AADL semantics. Finally, this architecture is tested for soundness, any 


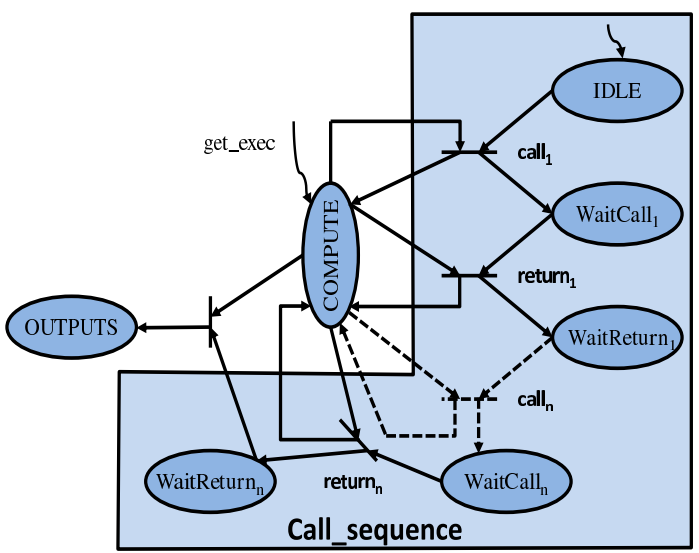

Fig. 7. BIP model for subprograms calls

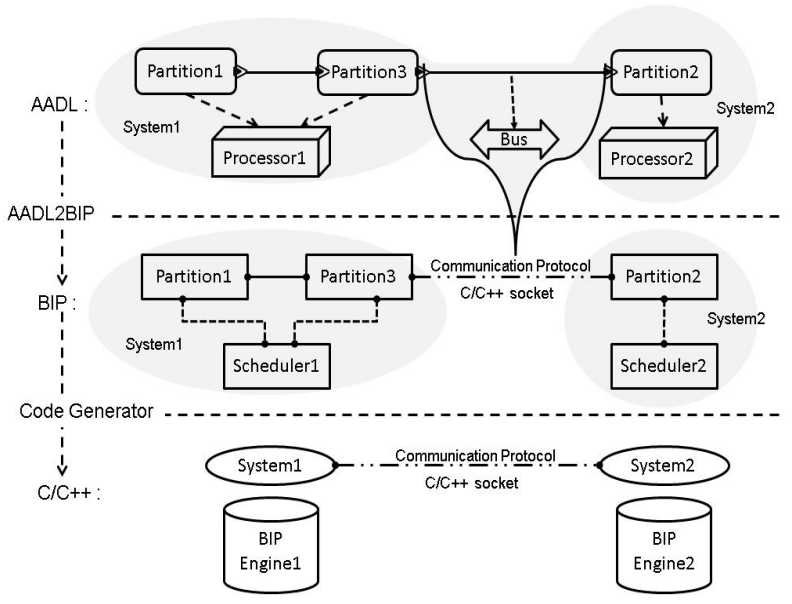

Fig. 8. Deployment

mismatch in the application is reported by the analysis BIP tool chain.

AADL is expressive enough to detail the deployment view of the application: threads, processors, buses, threads on each process; properties refine the type of tasks (periodicity, priority), and their associated implementation. We defined our distribution model as a set of sender/receiver. It is supported by an AADL architectural model that defines the location of each system and the payload of the message exchanged as a thread-port name plus possible additional data.

Figure 8 shows the steps for generating from a distributed AADL system's description an executable distributed application as follows:

(1) Identify each system and a connector's mapped to the bus.

(2) Generate for each AADL system its corresponding description in BIP, and for each connector's mapped to the bus a communication protocol.

(3) Compile BIP system and generate an executable for each system with communication protocol.

(4) Run and debug the distributed application.

Our protocol supports communication between two or more computers. It provides a full-duplex communication channel between processes that do not necessarily run on the same computer. We consider channels for data exchange among multiple threads in one or more processes are managed by the BIP Engine, if processes are running on one computer. Otherwise, if processes are running on different computers connected by a network, we use a network communication protocol. Before sending data through network to a server, we initially convert into encoded version before being transported (suitable for network transfer). After receiving data (Server side), it can be converted back.

Most network communication protocols use the client server model. These terms refer to the two machines which will be communicating with each other. One of the two machines, the client, connects to the other machine, the server, typically to make a request for information. Notice that the client needs to know of the existence of and the address of the server, but the server does not need to know the address of the client prior to the connection being established.

Our protocol uses sockets. Sockets are associated with the concept of network communication in the form of client-server programming; a pair of processes of which one will be a client and one a server. The client process will send requests to the server. Of course, when creating a socket, we have to specify the type of communication that will be needed, since different modes of communication require different protocols.

The generated BIP code provides a framework that will directly call user code when necessary. This allows a rapid and flexible design of the distributed system and does not restrict the user implementations.

\section{CASE STUDY}

\subsection{Precision Time Protocol}

The Precision Time Protocol (PTP) [16] is a time-transfer protocol defined in the IEEE 1588-2002 standard that allows precise synchronization of networks (e.g., Ethernet). The goal of this protocol is to have a set of slave devices determine the offset between time measurements on their clocks and time measurements on a master device.

In the protocol, the master device periodically launches an exchange of messages with slave devices to help each slave clock recompute the offset between its clock and the master's clock. This offset will drift with time, and so these periodic exchanges mitigate the impact of this drift on clock synchronization.

One assumption is that this exchange of messages happens over a period of time so small that this offset can safely be considered constant. Another assumption is that the transit time of a message going from the master to a slave is equal to the transit time of a message going from the slave to the master. Finally, it is assumed that both the master and slave can measure the time they send or receive a message. The degree to which these assumptions are enforced in an application regulate the accuracy of the offset measured at a slave device.

Synchronization messages are passed every two seconds to keep network resource usage at a minimum. Figure 9 shows the communication steps between the master and slave as follows :

(1) In the so called Sync-Message, the master sends its current time to the slave where a time stamp is generated exactly when the message is received.

(2) The master then sends a follow-up message to the slave with the exact time when the original sync-message had been sent. The slave then corrects its local real-time clock to the clock of the master. 


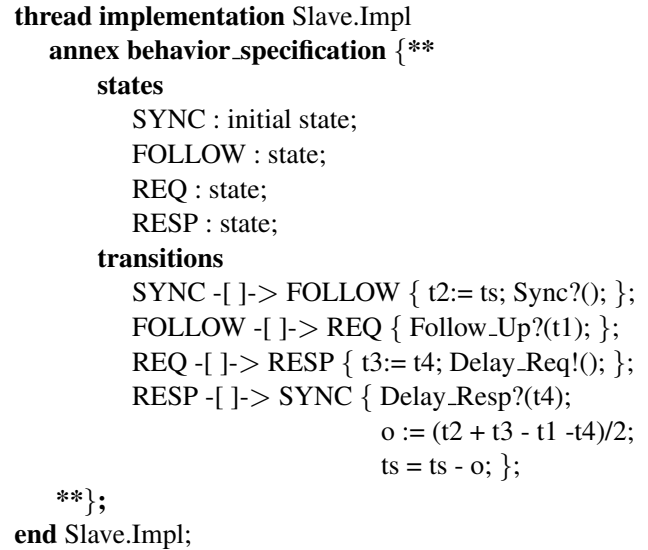

Fig. 13. Behavioral Specification of Slave

Figure 14 shows the software and hardware view of our case study. This model gathers typical elements from distributed systems, with a set of periodic tasks devoted to the processing of incoming orders. These two entities work at the same rates and should all respect their deadlines.

The software part represents how the processing is distributed onto different entities (threads) and gathered as AADL processes to form partitions. The next step is to map this part onto a physical hardware, so that processor resources can be associated to each process. The graphical representation of the deployment view of the system shows the global architecture of the application (number of processes and their mapping to hardware components). It indicates that each process is bound to a specific processor and how the communication between processors occurs, using different buses. In this case we use one bus that binds connections.

These two parts are expressed using the same modeling notation. They can be merged to form the complete system: interacting entities in the software part represent the processing logic of the system, whereas the hardware part completes the system deployment information by allocating resources.

Our PTP case study is built by creating software component and mapping entities onto a hardware architecture. The flexibility of AADL allows us to partially define components and use them in other components. This is very useful during the first steps of prototyping where every detail of the system is not yet clear. Details can be added to these components either by means of AADL properties or by component extension, without having to redefine all other components. PTP can be applied to more than one slave, in our case we consider one slave to keep the architecture clear.

\subsection{Validation}

The AADL model of the Precision Time Protocol System is transformed into BIP automatically by using our AADL to BIP translation tool. The generation of BIP code helps us to rapidly prototype the PTP case study and make it to a distributed application using our communication protocol between each partition. The prototype helped us to analyze the case study in a native platform in order to easily debug and evaluate it before running it on an embedded platform.

The separation between software and hardware in AADL allows the programmer to model all the software parts of his application and test it with a native platform (generally a PC). If the tests are

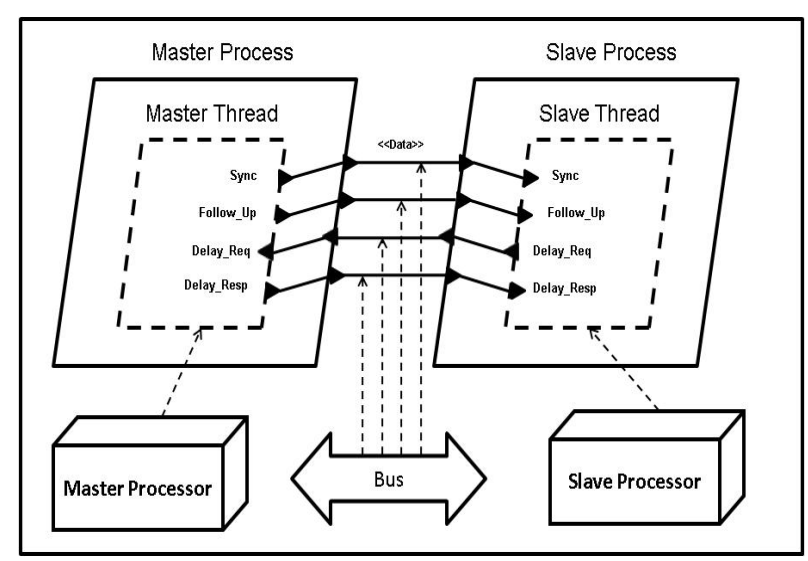

Fig. 14. Precision Time Protocol System

\begin{tabular}{|c|c|c|c|}
\hline \multirow{2}{*}{} & \multirow{2}{*}{ AADL } & \multicolumn{2}{|c|}{ BIP } \\
\cline { 3 - 4 } & & Master & Slave \\
\hline Components & 10 & 6 & 6 \\
\hline Connectors & 12 & 12 & 12 \\
\hline Lines of code & 200 & 170 & 150 \\
\hline
\end{tabular}

Fig. 15. Comparison between AADL \& BIP

successful, the same software part can be reused with the actual hardware AADL.

In the PTP case study, we generate for each AADL partition mapped to the processor, its corresponding description in BIP, and for each connection mapped to the bus a network communication protocol (sender/receiver). We compile BIP partitions and we generate an executable model. Then, we put every executable in the native platform (PC). First, we launch a receiver executable and then the sender executable. When the network protocol communication is initialized between the sender and receiver, the exchange of data is started.

Once the executable model has been launched, interactive simulation and debugging is useful for understanding the working of the distributed application. This helped us to verify each interaction step by step, to know which state or port is activated, and to see the value of data received/sender.

Figure 15 summarizes the size of lines of code, number of components and connectors in AADL and respectively the BIP code for the PTP case study. We split the BIP in two parts because we generate for each Master or Slave components a corresponding BIP description system.

In addition, we use BIP observer to express and check requirements. Observers allow us to express in a much simple manner most safety requirements. We apply this technique to verify two properties:

- Thread deadline: If the execution time of a thread exceeds its deadline the observer moves to an error state.

- Synchronisation between the Master and Slave: we use the BIP atomic component named Observer as shown in Figure 16 to verify the communication media. The role of this component is to control the communication between the Master and Slave components. The Observer component contains states to enforce the synchronization before moving to the next one. The initial state of the Observer is M_SYNC, when the Master thread is ready, the synchronization can take place through an interaction on the 
port $M_{-} S y n c$. In the state $S_{-} S Y N C$, the Observer component are waiting the synchronization with the Slave thread through an interaction on the port $S \_S y n c$. For example, if the matser send Follow_Up message and send a Delay_resp message without receiving the Delay_Req message the Observer moves to an error state.

These analysis allow to fully assess system viability, to refine and to correct the behavior of a system.

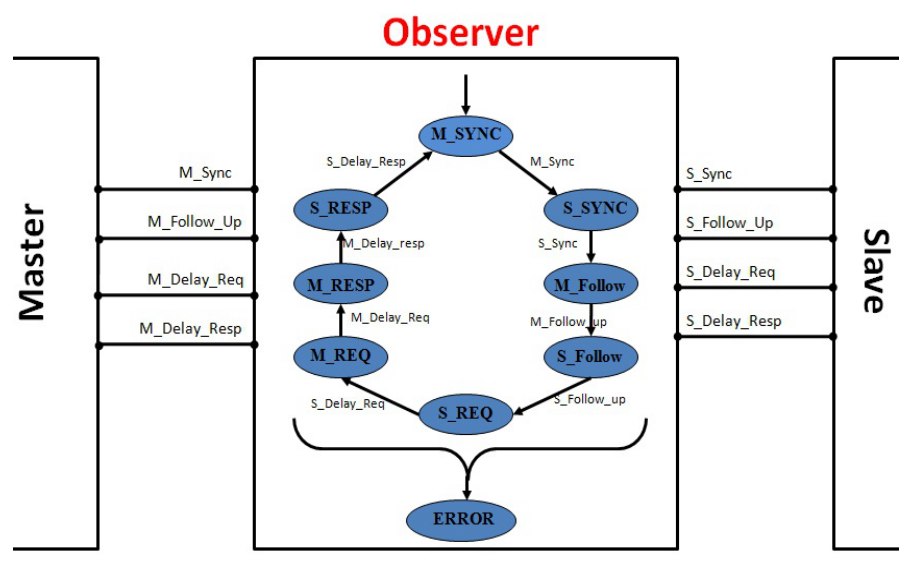

Fig. 16. Observer model in BIP

\section{CONCLUSION}

Prototyping distributed applications can be extremely useful in evaluating a design, and also in understanding the effect of different parameters on the performance of an application. We selected the Architecture Analysis and Design Language that provide adequate syntax and semantics to express and support distributed embedded systems. In this paper, we proposed a general methodology for translating AADL thread component depending on the thread implementation into the BIP language. We proposed also a general methodology for prototyping distributed applications using the Precision Time Protocol (PTP). In addition, we modeled and translated the case study from AADL into BIP. The executable application is tested and analyzed for soundness, any mismatch in the application is reported by the analysis BIP tool chain.

In the future we are working on the real-time clocks. This will allow real-time distributed algorithms to be implemented, and timing properties to be studied.

\section{REFERENCES}

[1] Annex Behavior Specification SAE AS5506.

[2] SAE. Architecture Analysis \& Design Language (standard SAE AS5506), September 2004, available at http://www.sae.org.

[3] SEI. Open Source AADL Tool Environment. http://la.sei.cmu.edu/aadlinfosite/ OpenSourceAADLToolEnvironment.html.

[4] TOPCASED: http://www.topcased.org/.

[5] A. Basu, S. Bensalem, M. Gallien, F. Ingrand, C. Lesire, T.H. Nguyen, and J. Sifakis. Incremental component-based construction and verification of a robotic system. In Proceedings of ECAI'08, Patras, Greece, 2008.
[6] A. Basu, M. Bozga, and J. Sifakis. Modeling heterogeneous real-time components in bip. In Proceedings of SEFM '06, Pune, India, pages 3-12. IEEE Computer Society, 2006.

[7] A. Basu, L. Mounier, M. Poulhiès, J. Pulou, and J. Sifakis. Using bip for modeling and verification of networked systems - a case study on tinyos-based networks. In Proceedings of NCA'07, Cambridge, MA USA, pages 257-260, 2007.

[8] S. Bensalem, M. Bozga, J. Sifakis, and T.H. Nguyen. Compositional verification for component-based systems and application. In Proceedings of ATVA'08, Seoul, South Korea, 2008.

[9] M. Bozga, J-C. Fernandez, A. Kerbrat, and L. Mounier. Protocol verification with the aldebaran toolset. STTT, 1:166-183, 1997.

[10] M. Bozga, S. Graf, Il. Ober, Iul. Ober, and J. Sifakis. The if toolset. In Proceedings of SFM'04, Bertinoro, Italy, volume 3185 of $L N C S$, pages 237-267.

[11] Mohamed Yassin Chkouri and Marius Bozga. Deterministic data flow communication in aadl. In Proceedings of the 2009 International Conference on Embedded Software and Systems, ICESS '09, pages 93-100. IEEE Computer Society, 2009.

[12] M.Y. Chkouri. Modeling of real-time embedded systems using $A A D L$ for the automatic generation of applications formally verified. PhD thesis, VERIMAG- University Joseph Fourier, 2010.

[13] M.Y. Chkouri, A. Robert, M. Bozga, and J. Sifakis. Translating AADL into BIP - Application to the Verification of RealTime Systems. In Models in Software Engineering: Workshops and Symposia at MODELS 2008, Toulouse, France, September 28 - October 3, 2008., pages 5-19.

[14] J. Sifakis G. Gossler. Composition for component-based modeling. Science of Computer Programming, 55:161-183, March 2005

[15] J. Hugues, B. Zalila, L. Pautet, and F. Kordon. Rapid Prototyping of Distributed Real-Time Embedded Systems Using the AADL and Ocarina. In Proceedings of the 18th IEEE International Workshop on Rapid System Prototyping (RSP'07), pages 106-112, Porto Alegre, Brazil, May 2007. IEEE Computer Society Press.

[16] Kang Lee and John Eidson. Ieee-1588 standard for a precision clock synchronization protocol for networked measurement and control systems. In In 34 th Annual Precise Time and Time Interval (PTTI) Meeting, pages 98-105, 2002.

[17] M. Poulhiès, J. Pulou, C. Rippert, and J. Sifakis. A methodology and supporting tools for the development of componentbased embedded systems. In 13th Monterey Workshop, Paris, France, volume 4888 of LNCS, pages 75-96, 2006.

[18] T. Vergnaud, J. Hugues, L. Pautet, and F. Kordon. PolyORB: a schizophrenic middleware to build versatile reliable distributed applications. In Proceedings of the 9th International Conference on Reliable Software Techologies AdaEurope 2004, volume LNCS 3063, pages 106 - 119, Palma de Mallorca, Spain, Jun. 